\title{
PRECISIONES SOBRE UN ARTÍCULO NOMENCLATURAL ACERCA DE LA VALIDEZ "TAXONÓMICA" DE IBERELLUS COMPANYONII (ALERON, 1837) RECIENTEMENTE PUBLICADO (MOLLUSCA, PULMONATA, HELICIDAE)
}

\author{
M. A. Alonso-Zarazaga
}

\begin{abstract}
RESUMEN
Se comenta, a la luz de una correcta interpretación del Código Internacional de Nomenclatura Zoológica, la propuesta de Forés (2003) de considerar Helix companyonii Aleron, 1837 como un nombre disponible para la Nomenclatura Zoológica. Se demuestra que dicho nombre no está disponible y que el nombre válido del taxon implicado es Iberellus pyrenaicus (Rossmässler, 1839), basado en Helix hispanica v. pyrenaica Rossmässler, 1839.

Palabras clave: Mollusca, Helicidae, Iberellus, Helix, nomenclatura, disponibilidad, validez.
\end{abstract}

\section{ABSTRACT}

Comments on a recently published nomenclatural article on the "taxonomic" validity of Iberellus compayonii (Aleron, 1837) (Mollusca, Pulmonata, Helicidae)

In the light of a correct interpretation of the International Code of Zoological Nomenclature, Forés's proposal (2003) of considering Helix companyonii Aleron, 1837 as an available name for Zoological Nomenclature is discussed. It is demonstrated that this name is not available and that the valid name for the implied taxon is Iberellus pyrenaicus (Rossmässler, 1839), based on Helix hispanica v. pyrenaica Rossmässler, 1839.

Key words: Mollusca, Helicidae, Iberellus, Helix, nomenclature, availability, validity.

\section{Introducción}

Recientemente, Forés (2003) ha publicado un largo artículo sobre la nomenclatura de una especie ibérica de Helicidae, en el que, interpretando de manera inadecuada el Código Internacional de Nomenclatura Zoológica, llega a unas conclusiones erróneas. El trabajo presenta la información de forma prolija, ilustrando todas las obras previas, de un modo desproporcionado al problema que se plantea, para cuya solución se precisa bastante menos información de la presentada. Como miembro de la Comisión Internacional de Nomenclatura Zoológica, este tipo de publicaciones, en que el Código Internacional de Nomenclatura Zoológica es utilizado de una manera un tanto personal e ina-

* Depto. de Biodiversidad y Biología Evolutiva, Museo Nacional de Ciencias Naturales - CSIC, José Gutiérrez Abascal, 2. E28006 Madrid 
decuada, me preocupa, lo que ha provocado las líneas que siguen, en las que se han respetado las grafías originales de la época, con algunos nombres específicos con inicial mayúscula. Para la bibliografía citada, consúltese el trabajo original.

\section{Exposición}

La intención de Forés (2003) es demostrar que el nombre correcto de una especie de Helicidae presente en Cataluña, el Archipiélago Balear y el departamento francés de Pyrénées-Orientales es Iberellus companyonii, basado en Helix companyonii Aleron, 1837, descrita, según el autor, mediante una referencia en un trabajo de Companyo (1837), para lo cual realiza un intensivo análisis histórico con profusión de copias de trabajos originales, muchos de los cuales no vienen al caso de la cuestión tratada. Ya el título del trabajo comienza confundiendo la validez taxonómica con la nomenclatural.

En la p. 142, el autor dice: “... aunque Rossmässler (1839) la considera una variedad de la H. hispanica Partsch...". A juzgar por las mismas páginas figuradas, en ningún momento Rossmässler dice esto, sino que en la pág. 11 indica Helix Campanyonii como sinónimo absoluto de su variedad pyrenaica de Helix hispanica, siendo esta variedad la descrita por él. En la figura 591 de la misma obra, el taxon nominal $H$. Campanyonii sufre la misma suerte, apareciendo entre paréntesis (simbolizando la sinonimia) detrás del nombre de la variedad. Es más, Rossmäsler no tiene certeza acerca de la autoría del taxon $H$. Campanyonii, al que en el texto hace seguir de un comentario "(cuiusn.?)", es decir, cuiusnam?, "¿perteneciente a quién?". Evidencia que el taxon nominal era de atribución dudosa en aquellas fechas. En la fig. 460 de su obra, Rossmäsler iconografía lo que él considera ser el taxon $H$. hispanica v. pyrenaica, pero no se le puede denominar como se hace en el pie de figura Iberellus balearicus, nombre que no vuelve a aparecer en la discusión, excepto como una posible subespecie.

En la p. 143, el autor comenta que el taxon $H$. hispanica var. pyrenaica Rossm. pudiera "ser considerado como disponible, si no fuera porque el actual Código Internacional de Nomenclatura Zoológica lo invalida". No especifica, sin embargo, el autor en qué puntos del articulado del Código se apoya para invalidar el taxon de Rossmässler. Unos párrafos antes reconoce que Rossmässler da una descripción sucinta de su Helix hispanica var. pyre- naica y que Helix Campanyonii (cuiusn.?) sec. Anton in litt. aparece como su sinónimo.

Asimismo, en la p. 144, el autor comenta que Mittre (1842) describe lo que él considera ser la misma especie (H. Minoricensis) "ignorando que estaba designando y describiendo la misma especie descubierta en los Pirineos Orientales por Aleron (1837) y Companyo (1837), ya que no pudo conocer los trabajos de Ortiz de Zárate (1946) y las indicaciones de otros autores que citaremos". Mittre describe honestamente una especie, mientras el autor omite dar referencia bibliográfica al trabajo de Aleron de 1837 (porque no existe, en todo caso es el de Companyo) y que, por tanto, Mittre no tenía por qué conocer.

Dupuy (1848, p. 120) cita este caracol como $H$. Companyonii Aleron. La fecha que da el autor está equivocada, pues, habiendo aparecido la obra del abate Dupuy en fascículos, cada uno tiene su propia fecha (Art. 21.5), y la página 120 corresponde al fascículo 2, de enero de 1848. Se podría fechar asimismo la lámina IV de la obra de Dupuy, que el autor nos ofrece en la figura 5i, de julio de 1847 , con lo que, caso de ser útil nomenclaturalmente, la figura tiene prioridad sobre el texto. En éste, la var. pyrenaica de Rossmässler de 1839 figura como sinónima del "Helix Companyonii Aleron, in Bulletin de la Soc. philom. de Perpignan, p. 91 et 98. [1837].... (1). " En la nota a pie de página, Dupuy resalta que ni Aleron ni Rossmässler han dado la diagnosis de esta especie, y que Rossmässler la ha llevado a variedad de $H$. Hispanica Partsch.

En la p. 151, el autor reconoce los hechos anteriormente comentados, y se basa en el Art. 12 de la Tercera Edición del Código Internacional de Nomenclatura Zoológica para dar por válido el nombre de la variedad de Rossmässler y, a la vez, el del sinónimo Helix Campanionii (sic). No obstante, la Tercera Edición del Código Internacional de Nomenclatura Zoológica dejó de ser válida el 31 de diciembre de 1999, pasando a ser efectiva desde el día 1 de enero de 2000 la Cuarta Edición (disponible con bastante anterioridad incluso en su traducción al castellano), por la cual el autor debió haberse regido en sus comentarios y disquisiciones (su manuscrito fue recibido en la revista el día 14 de octubre de 2002), y no por la Tercera. Por otro lado, el Art. 12 de la Tercera Edición es prácticamente idéntico al actualmente válido de la Cuarta, al que el autor recurre erróneamente para sancionar como válido algo que no lo es. El autor se equivoca, asimismo, al afirmar que la abreviatura "cuiusn.” es alemán, cuando es latín. 
Coincidiendo con el autor en que es posible y muy probable un error de Rossmässler, de Aleron por Anton, el autor encuentra que el nuevo Código (p. 152) "parece que deja disponible los dos nombres" (en la p. 143 ha mencionado la invalidez de la variedad de Rossmässler). Un nombre citado en sinonimia nunca está disponible, a menos que sea utilizado posteriormente como nombre válido en ciertos casos (Art. 11.6.1), como se discute más adelante.

Las citas posteriores de los diferentes usos de $H$. companyonii que menciona el autor son irrelevantes desde el punto de vista nomenclatural, así como el hecho de que den prioridad al taxon nominal $H$. Companyonii Aleron sobre $H$. hispanica var. pyrenaica Rossm., basándose fundamentalmente en una prioridad inexistente (1837 vs. 1839).

La inexistencia de descripción para la especie de Aleron es reconocida por varios de los malacólogos precedentes citados por el autor (p. ej. Hidalgo, 1890: pág. 174).

En la p. 183, el autor "deduce" (a partir de una carta que acredita la existencia de un "Tableau des mollusques du Département des PyrénéesOrientales" y fotografía del susodicho "Tableau", en el que se observan dos ejemplares, una etiqueta con el nombre $H$. companyonii y una descripción escrita a mano) la existencia de "una ilustración" y "una serie tipo".

\section{Conclusiones}

En el trabajo, cuya redacción y presentación son desproporcionadas a los objetivos que se persiguen (con páginas escaneadas de obras clásicas que no tienen importancia para la resolución de los problemas que plantea la nomenclatura de los táxones tratados, así como de interpretaciones sui-generis del Código Internacional de Nomenclatura Zoológica), el autor alcanza unas conclusiones erróneas.

Particularmente reveladora es la representación de la carta acreditativa de la existencia de "Le tableau des mollusques du Département de Pyrénées-Orientales" en la fig. 18 y del susodicho "Tableau" en la fig. 19. a y b, con una descripción manuscrita del taxon bajo el cartel del nombre y los dos ejemplares expuestos. No existe ningún artículo del Código que permita establecer una nomenclatura en función de los "certificados" que uno presente.

En las conclusiones, el autor cita el trabajo de Companyo (1837) del que deduce que la frase "Cette espèce se rapproche beaucoup de l'helix ser- pentina et ondulata" pude considerarse como una brevísima descripción o indicación. ¿En qué caracteres se basa la descripción, a qué referencia apunta la posible indicación? El autor nos remite entonces al comentario escrito, según él, por Aleron, bajo la etiqueta de los dos ejemplares del "Tableau" y que según él mismo dice "pude leer en mi visita al Muséum d'Histoire Naturelle de la Ciudad de Perpignan, donde está expuesto después de haberlo estado en la Société Philomatique".

En los párrafos siguientes a esta declaración, el autor prosigue con una serie de disquisiciones tendentes a demostrar que el "Tableau" es una descripción válida del taxon nominal en cuestión. Para ello comenta: "según el artículo 8 del Código Internacional de Nomenclatura Zoológica 4 [sic!] edición, la revista de 1837 de la Société Philomatique constituye una obra publicada por satisfacer los criterios del apartado 8.1 y no estar involucrada por los demás apartados...". Efectivamente, una revista como ésta es una obra publicada, a los efectos del Código. No tengo información de que en ella existan negaciones de la publicación (Art. 8.2) o de los nombres y actos incluidos (Art. 8.3.) o de que no fuese impresa sobre papel con tinta según métodos convencionales en la época (Art. 8.4), o de que haya sido suprimida (Art. 8.7).

Pero el hecho de que una obra esté publicada no confiere disponibilidad (o validez, conceptos diferentes que se deben separar netamente) a los táxones contenidos en ella. El autor declara que el asunto no está incluido en las disposiciones del Art. 9 ("Qué no constituye una obra publicada") y mezcla dos cosas distintas, por un lado la publicación de Companyo y por otra el "Tableau", como si fuesen la misma y única cosa. Además, concluye: "También debemos considerar que el cuadro en sí parece constituir una ilustración (Fig. 19a) puesto que dos individuos de la especie están en él, conjuntamente con otras especies y con sus nombres y comentarios correspondientes. Además como el cuadro estuvo y está, desde su origen, depositado y expuesto en una sociedad pública y después en un museo [...], también lo han estado los especímenes [...]."

La publicación de unos resultados científicos (inclusive una descripción de un taxon) y los ejemplares objeto de esa publicación son cosas diferentes que no se pueden mezclar. La disponibilidad de un nombre científico depende exclusivamente de la existencia de una publicación (Art. 11.1) y nunca de los ejemplares, que pueden haber desaparecido de la Naturaleza o de las colecciones. La publica- 
ción de Companyo (1837), según todos los autores, incluyendo el del trabajo que aquí se comenta, carece de descripción del taxon Helix companyonii. Este nombre no está disponible a efectos nomenclaturales a partir del trabajo de Companyo porque incumple el Art.12 para nombres propuestos antes de 1931, en particular el apartado 12.1 (carece de descripción o definición). Las palabras "Cette espèce se rapproche beaucoup de l'helix serpentina et ondulata" no pueden tomarse como una descripción o definición, pues son sólo una constatación de similitud morfológica que no revela diferencias diagnósticas del taxon en cuestión. No hay tampoco indicación (Art. 12.2) que pueda hacerlo disponible: referencia bibliográfica previa (Art. 12.2.1), referencia a índice de obra no binominal (Art. 12.2.2), ser un nombre de reemplazo (Art. 12.2.3), ser una descripción combinada de género y especie única nuevos (Art. 12.2.6), ser la denominación de una figura del taxon (Art. 12.2.7) o la descripción de la obra de un organismo (Art.12.2.8). En definitiva: no se puede atribuir un taxon disponible denominado Helix companyonii a Companyo o a Aleron, 1837: es un nomen nudum.

Lo único que une este trabajo de Companyo a especímenes tangibles es el "Tableau" al que se hace referencia. Queda por dilucidar, pues, si puede considerarse aquél como una "obra publicada", mientras que Forés (2003) intenta demostrar que se trata de una "ilustración" en el sentido del Código (y por lo tanto haría disponible el nombre por su mención en el trabajo de Companyo), para lo cual hace una larga disquisición, acudiendo inclusive al Diccionario Espasa, con la excusa de que el Código no da definición alguna de este término. Después de eso, señala: "Por ello la ubicación, el fin, el uso y el aspecto del cuadro de M. Aleron, según esta definición, pueden considerarse, en mi opinión [cursivas mías], como una ilustración. E igualmente, [...] el conjunto de la publicación de Companyo y el cuadro citado parecen constituir también una ilustración." A efectos del Código, una ilustración, como indicación, se refiere a una imagen publicada según los criterios del Código (Capítulo 3) o a una referencia bibliográfica de tal ilustración, siendo este último caso inadecuado a lo que aquí se considera. Por tanto, para el Código ese "Tableau" no es una obra publicada, ni como texto, ni como ilustración. Para que sea obra publicada el texto de la descripción escrito bajo la etiqueta, debiera haberse cumplido el Art. 8.1.3, y, a nuestro conocimiento, el "Tableau" es obra única e irrepetible, de la que no se han hecho nunca copias idénticas. No es óbice el ser texto manuscrito como pudiera pensarse, ya que existen obras basadas en planchas de texto manuscritas, de las cuales se hicieron largas tiradas, lo que no es el caso aquí. Con el nuevo Código, de todos modos, este sistema está rechazado (Art. 9.1). Para ser ilustración los ejemplares deben estar reproducidos en papel (Art. 8.4), lo cual no es el caso, porque se trata de conchas auténticas. Estamos, pues, ante el etiquetado de unos ejemplares de colección (no importa si los ejemplares están expuestos en un cuadro u ocultos en un cajón) cuyos nombres están excluidos de la disponibilidad nomenclatural por el Art. 12.3. El hecho de que estén a la vista del público no los convierte ipso facto ni en taxon publicado ni en tipos de tal.

El autor comenta las definiciones de la palabra "ilustración" de la Enciclopedia Espasa, pero de una forma, a mi juicio, parcial. Así leemos en la acepción b): "Estampa, grabado o dibujo que adorna un libro escrito", pero los objetos ilustrados no forman parte de la definición. O bien, en la c): "Publicación, comúnmente periódica, con láminas y dibujos [...]", es decir, algo sobre papel. El autor, sin embargo, se remite sólo a la acepción a): "Acción y efecto de ilustrar" y concluye con una definición: "Ilustración es la acción y efecto de aclarar un punto o materia con palabras, imágenes o de otro modo." Lo cierto es que, después de consumir 53 páginas de revista, no aclara nada acerca del nombre correcto del taxon en cuestión.

El resto del texto concluye (pág. 187) proponiendo como nombre válido de una especie Iberellus companyonii (Aleron, 1837), después de haber interpretado incorrectamente el Código. Nos informa de que el taxon nominal ha sido publicado conforme al Art. 11.1 (después de 1757), 11.2 (usando el alfabeto latino), 11.3 (uso del latín, griego u otros idiomas), 11.4 (uso de la nomenclatura binominal), 11.5 (tratamiento del nombre como válido para estar disponible) y 11.9 (condiciones para la disponibilidad de nombres de nivel especie), y que "parece cumplir [...] los requisitos del artículo 12" (aserción que ya se ha demostrado falsa). Además, señala "que cumple igualmente el espíritu de la ley contenida en el Código Internacional" la frase ya comentada, a la que ahora convierte, a más de descripción o indicación como hizo antes, en referencia: "Cette espèce se rapproche beaucoup de l'helix serpentina et ondulata". El motivo es que "el autor había consultado los textos de la época donde se contienen las definiciones de estas dos especies y las obras de clasificación que se citan en el "Rapport" ", apoyándose para ello en el Art. 12.2.7, que en ningún lugar dice que se haga disponible una especie 
consultando o citando la lista de obras usuales para determinar en una época dada.

Por lo demás, Forés (2003) toma por genitivo de Companyonus el término Companyonii, al que le sobra una i para serlo. Tampoco el nominativo Companyius daría Companyoi, sino más bien Companyii. De hecho, la latinización que hubiese dado Companyonii en el genitivo, habría sido en nominativo Companyonius. Fuera como fuese, la grafía original debe prevalecer.

Después de todo lo anterior, la resolución nomenclatural a la que se llega es errónea. De los datos presentados por Forés (2003) se deduce que la nomenclatura correcta debiera ser la siguiente:

Iberellus pyrenaicus (Rossmässler, 1839)

$=$ Helix companyonii Aleron in Companyo, 1837, nomen nudum

$=$ Helix hispanica $\mathrm{v}$. pyrenaica Rossmässler, 1839

$=$ Helix campanyonii Rossmässler, 1839, in synonymia

= Helix minoricensis Mittre, 1842

Queda por discernir si algún autor ha validado el nombre de Helix companyonii (o alguna de sus varias grafías) conforme a lo dispuesto por el Código. Aparentemente, el primero en hacerlo es Dupuy (1847) conforme a los datos suministrados por Forés (2003), de manera que el taxon nominal sería Helix companyonii Dupuy, 1847 (pl. IV, fig. 3 ), posterior a $H$. pyrenaica y a $H$. minoricensis.

Pudiera también considerarse que el taxon nominal $H$. campanyonii deba ser atribuido a Rossmässler, 1839 (Art. 50.7), por haber sido descrito originalmente en sinonimia y validado posteriormente al ser utilizado para un taxon válido (Art. 11.6.1). Pero el caso es que nadie, al parecer - al menos en los ejemplos ofrecidos por Forés (2003) — , ha denominado la especie usando este taxon nominal con este autor, sino que siempre se le ha llamado H. companyonii Aleron, 1837 (o variantes). Ambos táxones nominales difieren en una letra (cuando no en más por las variantes gráficas) y el nombre de $H$. campanyonii Rossmässler no puede ser modificado (Art. 32.3) sin incurrir en una enmienda injustificada (Art. 33.2), dado que no encuentro en la obra original de este autor evidencia de una errata (Art. 32.5.1). En esta tesitura, considero que el nombre $H$. campanyonii Rossmässler no ha sido utilizado antes de 1961 como nombre válido (Art. 11.6.1) y, por tanto, no está disponible.

Como conclusión final, y como miembro de la Comisión Internacional de Nomenclatura Zoológica, me permito sugerir que para la resolución de problemas nomenclaturales complejos y para una adecuada interpretación del Código se acuda al asesoramiento de dicha Comisión o de expertos en nomenclatura zoológica. Al menos, los comités editoriales de las revistas donde tales artículos vayan a ser publicados debieran hacerlo a través de la selección de revisores adecuados.

\section{AGRADECIMIENTOS}

Quisiera agradecer a los revisores de este artículo, los Dres. Sandro Minelli, Enrique Macpherson Mayol y José Templado González, sus múltiples sugerencias y comentarios, que han mejorado considerablemente la redacción original.

\section{Referencias}

ForÉS, M. 2003. Sobre la validez taxonómica [sic!] de Iberellus companyonii (Aleron, 1837) (Pulmonata: Helicidae). Bolletí de la Societat d'Història Natural de les Balears, [2002], 45: 137-189.
Recibido, 1-III-2004 Aceptado, 14-VI-2004 Publicado, 25-VIII-2004 